\title{
Electron Pair Localization Function (EPLF) for Density Functional Theory and $a b$ Initio Wave Function-Based Methods: A New Tool for Chemical Interpretation
}

\author{
Anthony Scemama, ${ }^{,+}{ }^{\dagger}$ Michel Caffarel, ${ }^{\dagger}$ Robin Chaudret, ${ }^{\dagger}$ and Jean-Philip Piquemal ${ }^{\ddagger}$ \\ ${ }^{\dagger}$ Laboratoire de Chimie et Physique Quantiques, CNRS-IRSAMC, Université de Toulouse, France \\ ${ }^{\ddagger}$ UPMC Univ Paris 06, UMR 7616, Laboratoire de Chimie Théorique, case courrier 137, 4 place Jussieu, F-75005, Paris, France, and \\ CNRS, UMR 7616, Laboratoire de Chimie Théorique, case courrier 137, 4 place Jussieu, F-75005, Paris, France
}

Supporting Information

ABSTRACT: We present a modified definition of the Electron Pair Localization Function (EPLF), initially defined within the framework of quantum Monte Carlo approaches [Scemama, A.; Caffarel, M.; Chaquin, P. J. Chem. Phys. 2004, 121, 1725] to be used in Density Functional Theories (DFT) and ab initio wave-function-based methods. This modified version of the EPLF-while keeping the same physical and chemical contents-is built to be analytically computable with standard wave functions or Kohn-Sham representations. It is illustrated that the EPLF defines a simple and powerful tool for chemical interpretation via selected applications including atomic and molecular closed-shell systems, $\sigma$ and $\pi$ bonds, radical and singlet open-shell systems, and molecules having a strong multiconfigurational character. Some applications of the EPLF are presented at various levels of theory and compared to Becke and Edgecombe's Electron Localization Function (ELF). Our open-source parallel software implementation of the EPLF opens the possibility of its use by a large community of chemists interested in the chemical interpretation of complex electronic structures.

\section{INTRODUCTION}

Nowadays, when dealing with theoretical chemical interpretation, quantum chemists rely on two main strategies. The first consists of the traditional direct interpretation of the wave function through its projection onto molecular orbitals (MO) or valence bond (VB) structures (the so-called Hilbert space partitioning). The second uses a geometrical direct-space description in order to partition the electronic density into domains within the ordinary $3 \mathrm{D}$ space. The design of such interpretative techniques, initiated by Daudel et al., ${ }^{1}$ was popularized by Bader, who introduced the Quantum Theory of Atoms in Molecules (QTAIM). ${ }^{2}$ Along with QTAIM, Bader introduced the concept of topological analysis, offering an atom-based partition of the molecular space grounded on the gradient dynamical system theory and using a local function, here the Laplacian of the electron density. Through the years, much effort has been devoted to the design of alternative local functions. For example, Becke and Edgebombe introduced the Electron Localization Function (ELF), ${ }^{3}$ offering access to chemically intuitive domains beyond atomic centers encompassing bonds, lone pairs, etc. Ever since, its usefulness has been demonstrated by Silvi and Savin, ${ }^{4}$ who extensively developed its topological analysis, although no partition of space is unique. ${ }^{5}$

The problem of getting an accurate description of chemical bonding gets more and more difficult as the complexity of the wave function goes beyond the single determinant approximation. ${ }^{6}$ Therefore, an additional natural orbital approximation was added to the ELF formalism ${ }^{7}$ to extend it to the correlated level, but its general applicability to any quantum chemical method is still subject to intense development. In that context, other methods were introduced such as the electron localizability indicator (ELI, see ref 8 and references therein), the analysis of electronic probability distributions, ${ }^{9,10}$ and the Electron Pair Localization Function (EPLF). ${ }^{11}$

In this work, we shall focus on this latter function, EPLF, whose main feature is giving direct access to the local (spatial) electronic correlations between spin-like and spin-unlike electronic pairs. EPLF was first introduced within the framework of quantum Monte Carlo (QMC) approaches where introducing simple and direct estimators of such local electronic correlations is particularly easy. In practice, it has been proposed to build an indicator - the electron pair localization function-based on a suitable combination of the average distances between an electron of a given spin located at point $\mathbf{r}$ and the closest spinlike and spin-unlike electrons. EPLF has been shown to be particularly interesting to get new insights into the nature of the pairing and localization of electrons and, particularly, to understand more deeply the role of the dynamical and nondynamical near-degeneracy correlation effects. ${ }^{12,13}$ From a fundamental point of view, such a result is not surprising, since the EPLF is actually related to the conditional probabilities of finding an electron at point $\mathbf{r}_{2}$ with spin $\sigma$ or $\bar{\sigma}$, knowing that an electron of a given spin $\sigma$ is located at some point $\mathbf{r}_{1}$. Indeed, having such quantities at our disposal is known to be sufficient to define an exact electronic structure theory (e.g., the exact exchangecorrelation energy of DFT can be in principle derived from such conditional probabilities, ;see, e.g., ref 14). The advantage of having defined the EPLF within a QMC computational scheme is that such a function can be easily calculated at various levels of approximation. Indeed, by generating QMC probability densities

Received: October 15, 2010

Published: February 02, 2011 
associated with various trial wave functions, the average distances between electrons and, thus, the EPLF function can be evaluated at the Hartree-Fock, DFT, CASSCF, CI, VB, etc. levels of approximation. It is also possible to evaluate the EPLF at the fixed-node diffusion Monte Carlo level, a particularly accurate QMC approximation recovering the major part of static and dynamical correlation effects, even if in some cases the quality of the fixed-node error is not so easy to assess, see, e.g., ref 15 . Besides these advantages, the main drawback of calculating the EPLF with QMC is that simulations need to be rather intensive to decrease sufficiently the statistical errors of the EPLF values at each point $r$ of the grid employed. Indeed, a minimal resolution is needed to distinguish the subtle changes in local properties.

In this work, we propose a modified form for the EPLF allowing its exact computation (no statistical error) for the standard wave functions of computational chemistry written as determinantal expansions built from molecular orbitals expressed in some Gaussian basis set. The approach can also be naturally applied to DFT calculations based on a Kohn-Sham density expressed in a determinantal form. As we shall see, the proposed modification of the EPLF does not alter its chemical content. Using this modified expression, the EPLF is much more rapid to compute since its calculation requires only the evaluation of monoelectronic integrals (see below). In particular, it avoids the use of Monte Carlo sampling, which can be rather CPU-intensive for large systems, opening the possibility to perform full topological analyses in the near future. Accordingly, once introduced into standard computational chemistry packages, we believe that the EPLF will become a very useful and powerful tool for chemical interpretation accessible to a wide community of chemists.

\section{EPLF: THE ORIGINAL DEFINITION}

In the original definition of the EPLF, ref 11, the motivation was to define a function of $\mathbb{R}^{3}$ measuring locally the electron pairing in a molecular system. To do that, the following definition of electron pairing was first introduced: An electron $i$ located at $\mathbf{r}_{i}$ is said to be paired to an electron $j$ located at $\mathbf{r}_{j}$ if electron $j$ is the closest electron to $i$. Having defined such a pairing, it has been proposed to define the amount of electron pairing at point $r$ in terms of a quantity inversely proportional to

$$
d(\mathbf{r})=\left\langle\Psi\left|\sum_{i=1, N} \delta\left(\mathbf{r}-\mathbf{r}_{i}\right) \min _{j \neq i} r_{i j}\right| \Psi\right\rangle
$$

where $d(\mathbf{r})$ can be interpreted as the average of the shortest electron-electron distance at $\mathbf{r}, \Psi\left(\mathbf{r}_{1}, \ldots, \mathbf{r}_{N}\right)$ being the $N$-electron wave function, and $r_{i j}=\left|\mathbf{r}_{i}-\mathbf{r}_{j}\right|$.

Two different types of electron pairs are to be defined: pairs of electrons having the same spin $(\sigma)$ and pairs of electrons with opposite spins $(\sigma, \bar{\sigma})$. Hence, two quantities need to be introduced:

$$
\begin{aligned}
& d_{\sigma \sigma}(\mathbf{r})=\left\langle\Psi\left|\sum_{i=1, N} \delta\left(\mathbf{r}-\mathbf{r}_{i}\right) \min _{j \neq i ; \sigma_{i}=\sigma_{j}} r_{i j}\right| \Psi\right\rangle \\
& d_{\sigma \bar{\sigma}}(\mathbf{r})=\left\langle\Psi\left|\sum_{i=1, N} \delta\left(\mathbf{r}-\mathbf{r}_{i}\right) \min _{j ; \sigma_{i} \neq \sigma_{j}} r_{i j}\right| \Psi\right\rangle
\end{aligned}
$$

The electron pair localization function is bound in the $[-1,1]$ interval and is defined as

$$
\operatorname{EPLF}(\mathbf{r})=\frac{d_{\sigma \sigma}(\mathbf{r})-d_{\sigma \bar{\sigma}}(\mathbf{r})}{d_{\sigma \sigma}(\mathbf{r})+d_{\sigma \bar{\sigma}}(\mathbf{r})}
$$

When the pairing of spin-unlike electrons is predominant, $d_{\sigma \sigma}(\mathbf{r})>$ $d_{\sigma o}(\mathbf{r})$ and $\operatorname{EPLF}(\mathbf{r})>0$. When the pairing of spin-like electrons is predominant, $d_{\sigma \sigma}(\mathbf{r})<d_{\sigma o}(\mathbf{r})$ and $\operatorname{EPLF}(\mathbf{r})<0$. When the electron pairing of spin-like and spin-unlike electrons is equivalent, $\operatorname{EPLF}(\mathbf{r}) \sim 0$.

This localization function does not depend on the type of wave function and can therefore measure electron pairing using any kind of representation: Hartree-Fock (HF), Kohn-Sham (KS), Configuration Interaction (CI), and Multi-Configurational Self-Consistent-Field (MCSCF) as well as Slater-Jastrow, Diffusion Monte Carlo (DMC), Hylleraas wave functions, etc. Due to the presence of the min function in the definitions of $d_{\sigma \sigma}(\mathbf{r})$ and $d_{\sigma o}(\mathbf{r})$, these quantities cannot be evaluated in an analytical way, and quantum Monte Carlo (QMC) approaches appear to be the most efficient way of computing the three-dimensional EPLF grids via a statistical sampling of $\sim \Psi^{2}\left(\mathbf{r}_{1}, \ldots, \mathbf{r}_{N}\right)$ in the case of Variational Monte Carlo (VMC)-type calculations or $\sim \Psi\left(\mathbf{r}_{1}, \ldots, \mathbf{r}_{N}\right)$ $\Phi_{0}\left(\mathbf{r}_{1}, \ldots, \mathbf{r}_{N}\right)\left(\Phi_{0}\right.$ fixed-node ground-state wave function) in the case of the more accurate Fixed-Node Diffusion Monte Carlo (FN-DMC)-type calculations ${ }^{11-13,16}$ (for a detailed presentation of these various versions of QMC approaches, see, e.g., ref 17).

\section{EPLF: A MODIFIED DEFINITION SUITABLE FOR DFT AND WAVE FUNCTION-BASED METHODS}

Following preliminary developments, ${ }^{18}$ we propose here to introduce a modified definition of the EPLF which-in contrast with the original definition - can now be analytically computable for standard wave functions of quantum chemistry, thus avoiding the need for statistical sampling. To do that, we propose to express the $\mathrm{min}$ function appearing in the average distances in terms of Gaussian functions. More precisely, we introduce the following exact representation:

$$
\min _{j \neq i} r_{i j}=\lim _{\gamma \rightarrow+\infty} \sqrt{-\frac{1}{\gamma} \ln f\left(\gamma ; r_{i j}\right)}
$$

with

$$
f\left(\gamma ; r_{i j}\right)=\sum_{j \neq i} \mathrm{e}^{-\gamma r_{i j}^{2}}
$$

Now, our basic approximation consists in replacing, for $\gamma$ large, the integrals

$$
\left\langle\Psi\left|\sum_{i=1}^{N} \delta\left(\mathbf{r}-\mathbf{r}_{i}\right)\left(\sqrt{-\frac{1}{\gamma} \ln f\left(\gamma ; r_{i j}\right)}\right)\right| \Psi\right\rangle
$$

appearing in eq 1 with

$$
\sqrt{-\frac{1}{\gamma} \ln \left\langle\Psi\left|\sum_{i=1}^{N} \delta\left(\mathbf{r}-\mathbf{r}_{i}\right) f\left(\gamma ; r_{i j}\right)\right| \Psi\right\rangle}
$$

The expectation values of the minimum distances are now given by

$$
\begin{aligned}
& d_{\sigma \sigma}(\mathbf{r}) \underset{\gamma \text { large }}{\sim} \sqrt{-\frac{1}{\gamma} \ln \bar{f}_{\sigma \sigma}(\gamma ; \mathbf{r})} \\
& d_{\sigma \bar{\sigma}}(\mathbf{r}) \underset{\gamma \text { large }}{\sim} \sqrt{-\frac{1}{\gamma} \ln \bar{f}_{\sigma \bar{\sigma}}(\gamma ; \mathbf{r})}
\end{aligned}
$$


with the two-electron integrals:

$$
\begin{gathered}
\bar{f}_{\sigma \sigma}(\gamma ; \mathbf{r})=\left\langle\Psi\left|\sum_{i=1}^{N} \delta\left(\mathbf{r}-\mathbf{r}_{i}\right) \sum_{j \neq i ; \sigma_{i}=\sigma_{j}}^{N} \mathrm{e}^{-\gamma\left|\mathbf{r}_{i}-\mathbf{r}_{j}\right|^{2}}\right| \Psi\right\rangle \\
\bar{f}_{\sigma \bar{\sigma}}(\gamma ; \mathbf{r})=\left\langle\Psi\left|\sum_{i=1}^{N} \delta\left(\mathbf{r}-\mathbf{r}_{i}\right) \sum_{j ; \sigma_{i} \neq \sigma_{j}}^{N} \mathrm{e}^{-\gamma\left|\mathbf{r}_{i}-\mathbf{r}_{j}\right|^{2}}\right| \Psi\right\rangle
\end{gathered}
$$

When the wave function $\Psi$ has a standard form (sum of determinants built from molecular integrals $\phi$ 's), such integrals can be easily obtained in terms of the following elementary contributions:

$$
\phi_{i}(\mathbf{r}) \phi_{k}(\mathbf{r}) \int \mathrm{d} \mathbf{r}^{\prime} \phi_{j}\left(\mathbf{r}^{\prime}\right) \phi_{l}\left(\mathbf{r}^{\prime}\right) \mathrm{e}^{-\gamma\left|\mathbf{r}-\mathbf{r}^{\prime}\right|^{2}}
$$

which in turn can be evaluated as generalized overlap integrals.

Let us now discuss our basic approximation consisting in going from eq 7 to eq 8 . This approximation can be written in a more compact way as

$$
\frac{\langle\sqrt{-\ln f}\rangle}{\sqrt{-\ln \langle f\rangle}} \underset{\gamma \text { large }}{\sim} 1
$$

where the symbol $\langle Q\rangle$ denotes the integration of $Q \Psi^{2}$ over all particle coordinates except the $i$ th one. For a given electronic configuration $\left(\mathbf{r}_{1}, \ldots, \mathbf{r}_{N}\right)$ and $\gamma$ large enough, $f$ is dominated by a single exponential, namely, $\mathrm{e}^{-\gamma\left|\mathbf{r}_{i}-\mathbf{r}_{\text {jmin }}\right|^{2}}$, where $\left|\mathbf{r}_{i}-\mathbf{r}_{j_{\min }}\right|$ is the distance between the reference electron $i$ located at $\mathbf{r}_{i}$ and the closest electron labeled $j_{\text {min }}$. The validity of our basic approximation is directly related to the amount of fluctuations of the quantity $f$ when various electronic configurations are considered. Note that for a given electron $i$, the distance $\left|\mathbf{r}_{i}-\mathbf{r}_{j}\right|$ can vary a lot, but it is much less the case for $\left|\mathbf{r}_{i}-\mathbf{r}_{j_{\min }}\right|$, where the electron number $j_{\min }$ can be different from one configuration to another. When these fluctuations are small, the ratio in eq 14 is close to 1 and the approximation is of good quality. To see what happens for larger fluctuations, let us write

$$
f=f_{\min }+\delta f
$$

A simple calculation leads to

$$
\frac{\langle\sqrt{-\ln f}\rangle}{\sqrt{-\ln \langle f\rangle}}=1+O\left[(\delta f)^{2}\right]
$$

showing that at first order in the fluctuations the ratio is still equal to 1 , illustrating the validity of our approximation.

A last point to discuss is the value of $\gamma$ to be chosen in practice. Because of our approximation, the limit $\gamma \rightarrow+\infty$ cannot be taken since the ratio in eq 14 goes to zero. ${ }^{19}$ Therefore, the value of $\gamma$ has to be large enough to discriminate between the closest electron located at $r_{\text {jmin }}$ from the other ones located at larger distances of electron $i$, while staying in the regime where the ratio in eq 14 stays close to one. We have found that a value of $\gamma$ depending on $\mathbf{r}$ and chosen on physical grounds allows systematic recovery of the essential features of the EPLF images calculated with QMC, that is to say, with the exact expression of the min function. To be effective, the discrimination of the closest electron with the other ones must be properly implemented. To do that, the value of $\gamma$ is adapted to keep the leading

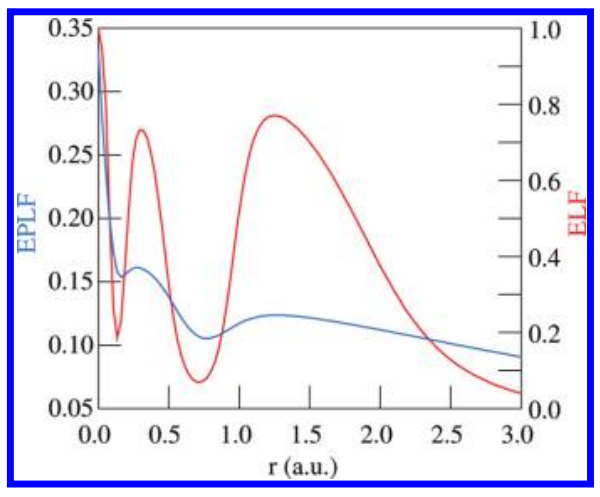

Figure 1. ELF and EPLF radial values for the argon atom as a function of the distance to the nucleus.

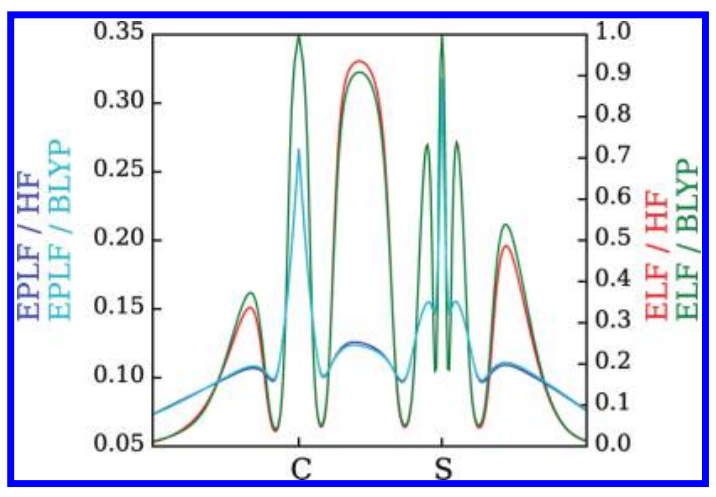

Figure 2. ELF and EPLF values in the $\mathrm{CH}_{3} \mathrm{~S}^{-}$anion along the $\mathrm{C}-\mathrm{S}$ axis computed using a Hartree-Fock and a BLYP determinant.

exponential $\mathrm{e}^{-\gamma\left|\mathbf{r}_{i}-\mathbf{r}_{\text {min }}\right|^{2}}$ significantly larger than the subleading exponential $\left.\mathrm{e}^{-\gamma \mid \mathbf{r}_{i}-\mathbf{r}_{\text {next }}-\text { min }}\right|^{2}$ associated with the second closest electron $j_{\text {next }- \text { min }}$. First, we define a sphere $\Omega\left(\mathbf{r}_{i}\right)$ centered on $\mathbf{r}_{i}$ with a radius $d_{\Omega}\left(\mathbf{r}_{i}\right)$. Then, locally, we represent our system made of the electron located at $\mathbf{r}_{i}$ and its two closest neighbors by a model system of three independent particles. If one calculates the probability of finding all three particles inside the sphere, one finds

$$
P_{\Omega}\left(\mathbf{r}_{i}\right)=\left(\frac{1}{3} \int_{\Omega\left(\mathbf{r}_{i}\right)} \mathrm{d} \mathbf{r} \rho(\mathbf{r})\right)^{3}
$$

If the density $\rho(\mathbf{r})$ is supposed as constant and equal to $\rho\left(\mathbf{r}_{i}\right)$, the radius $d_{\Omega}\left(\mathbf{r}_{i}\right)$ of the sphere can be set such that $P_{\Omega}$ is equal to a fixed value:

$$
d_{\Omega}\left(\mathbf{r}_{i}\right)=\left(\frac{4 \pi}{9} P_{\Omega}{ }^{-1 / 3} \rho\left(\mathbf{r}_{i}\right)\right)^{-1 / 3}
$$

Then, $\gamma\left(\mathbf{r}_{i}\right)$ is chosen in order to set a constant ratio $\kappa$ between the width of $\mathrm{e}^{-\gamma r_{i j}{ }^{2}}$ and the radius of the sphere

$$
\kappa=\sqrt{2 \gamma\left(\mathbf{r}_{i}\right)} d_{\Omega}\left(\mathbf{r}_{i}\right)
$$

We obtain an expression of $\gamma\left(\mathbf{r}_{i}\right)$ which depends on the electron density:

$$
\gamma\left(\mathbf{r}_{i}\right)=\frac{\kappa^{2}}{2}\left(\frac{4 \pi}{9} P_{\Omega}{ }^{-1 / 3} \rho\left(\mathbf{r}_{i}\right)\right)^{2 / 3}
$$

In our simulations, we have found that the EPLF images obtained with QMC are properly recovered using $P_{\Omega}=0.001$ and $\kappa=50$. 


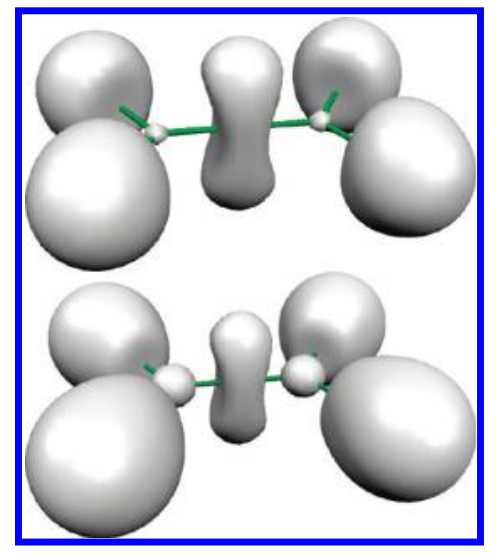

Figure 3. $\mathrm{ELF}=0.75$ (top) and EPLF $=0.12$ (bottom) isosurfaces of the ethylene molecule.

\section{SOME APPLICATIONS}

As discussed previously, the modified form of the EPLF presented here is aimed at providing the same chemical information as the original QMC-based EPLF scheme, but without the statistical noise inherited from the QMC approach. Therefore, the interested reader can refer to existing recent publications that deal with the QMC-EPLF analysis of covalent, ionic, and multicenter bonds. ${ }^{11-13,16,20}$ We focus in this section on some illustrative applications highlighting the specific capabilities of the EPLF as compared to Becke and Edgecombe's ELF.

4.1. Closed-Shell Single-Determinant Systems. A first natural example to look at is the case of a closed-shell atom described at the Hartree-Fock (HF) level. Using Dunning's cc-pVDZ atomic basis set, ${ }^{21}$ the radial values of the EPLF and ELF for the argon atom are displayed in Figure 1. It is noted that both functions display three maximum values corresponding to the $n=1, n=2$, and $n=3$ values of the principal quantum number. Furthermore, these maxima are essentially located at the same place. The gross features of the atomic shell structure are thus described in a similar way by both approaches. However, there is also a striking difference: The magnitudes of the two secondary maxima corresponding to the two most external shells are essentially identical in the ELF case but very different for the EPLF, where the outermost one is much smaller. Note that having such a difference is not surprising since EPLF is, in contrast with ELF, directly connected to electron pairing. The pairing of antiparallel electrons is likely to be the strongest in the first shell, weaker in the second shell, and the weakest in the most diffuse third shell.

EPLF and ELF were computed for the $\mathrm{CH}_{3} \mathrm{~S}^{-}$methanethiolate anion, using a Hartree-Fock determinant and a KohnSham determinant. The $6-31^{+2} \mathrm{G}^{* *}$ atomic basis set ${ }^{22,23}$ was used for both determinants, and the BLYP functional ${ }^{24,25}$ was used for the DFT calculation. Figure 2 compares the one-dimensional plots of the EPLF and ELF along the $\mathrm{C}-\mathrm{S}$ axis of the tetrahedral $\mathrm{CH}_{3} \mathrm{~S}^{-}$. As for the argon atom, the topologies of the EPLF and ELF functions are comparable for both the Hartree-Fock and the Kohn-Sham determinants. Going from the Hartree-Fock to the BLYP level, the values of the ELF are essentially the same in the core domains, become slightly smaller in the $\mathrm{C}-\mathrm{S}$ bonding region, and become slightly larger on the rest of the $\mathrm{C}-\mathrm{S}$ axis. As the EPLF exhibits the same trend, we conclude that for closed-shell single determinants the EPLF and ELF give qualitatively similar results.

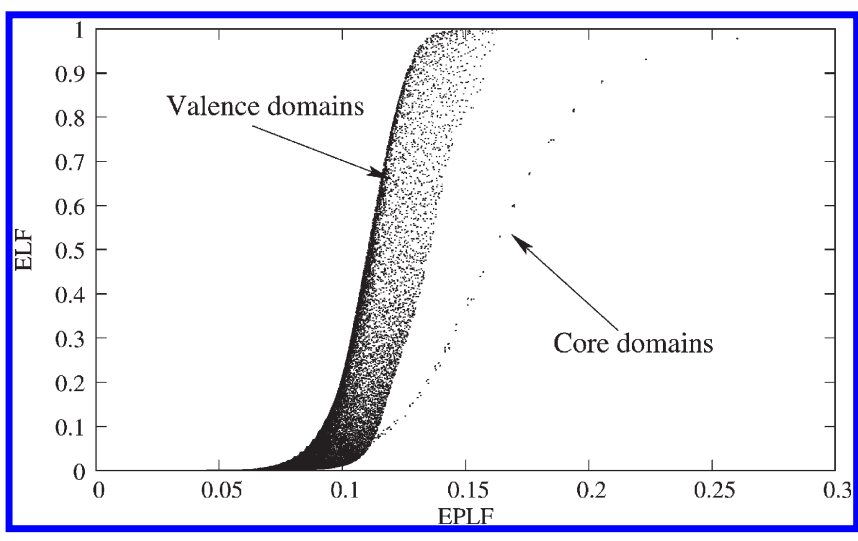

Figure 4. Correlation between the ELF and the EPLF in the ethylene molecule.

The ELF and EPLF were computed for the ethylene molecule using a HF/cc-pVDZ wave function. The isosurfaces $\mathrm{ELF}=0.75$ and $\mathrm{EPLF}=0.12$ are represented in Figure 3. These images are qualitatively similar, even if the core domains seem to be larger using the EPLF. This is due to the fact that the EPLF values are higher in the first atomic shells (as in the argon example), while the ELF has more comparable values among the shells.

To have a more quantitative visualization of the similarities and differences between the ELF and the EPLF, a correlation plot relating the values of both functions is presented in Figure 4. Three different regimes can be observed. First, a regime corresponding to the core domain where the EPLF takes its larger values. In this region, an almost perfect one-to-one correspondence is observed, thus illustrating the similarity between both localization functions. In contrast, in the valence region where the (EPLF,ELF) points are scattered, it seems to be no longer true. In fact, this is not really the case since the majority of points are found to be almost aligned along the left side of the envelope of points. To illustrate this, the median line (same number of points on each side) is represented. Finally, a last regime corresponding to the region where the ELF and EPLF values are small (say, ELF smaller than 0.05) can be defined. In such a regime, the two localization functions turn out to be fully decorrelated. However, the underlying configurations correspond to regions in space where the electronic densities are (very) small, and this case is not of great chemical interest. As a conclusion, in all chemically interesting regimes, the correlation between ELF and EPLF is high. We have found that such a conclusion is valid not only for this case but also for all molecules described by a closed-shell single determinant wave function. In this case, the qualitative information that can be obtained from an ELF and an EPLF calculation is essentially the same. This can be understood by noting that for a closed-shell monoconfigurational wave function the $\alpha$ electrons are independent from the $\beta$ electrons, so localizing electrons is essentially equivalent to localizing antiparallel electron pairs.

4.2. Open-Shell Hartree-Fock. A wave function for the $\mathrm{HC}_{2}{ }^{\bullet}$ radical was obtained at the restricted open-shell HartreeFock level (ROHF), using the cc-pVDZ atomic basis set. Both ELF and EPLF were computed, and the results are displayed in Figure 5. This example points out the main difference between ELF and EPLF: the localization region of the unpaired electron exhibits a maximum for ELF (high electron localization) and a minimum for EPLF (low electron pairing). EPLF can identify 


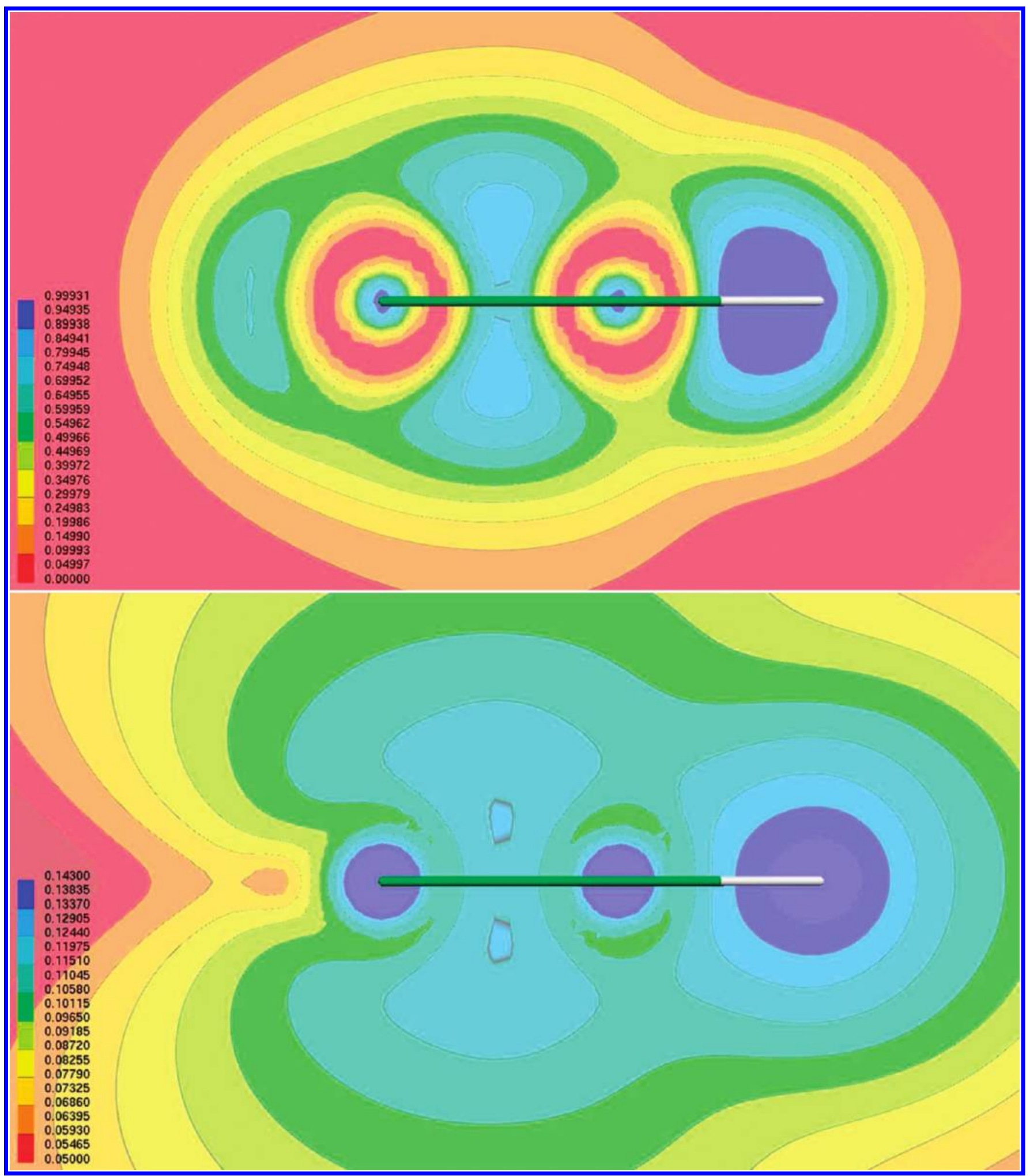

Figure 5. ELF (top) and EPLF (bottom) contour plots of the $\mathrm{HC}_{2}{ }^{\bullet}$ radical in the molecular plane. Red values are the lowest and blue values are the highest.

clearly domains of electron pairing (lone pairs, core domains, and bonds), and it can additionally characterize localized unpaired electrons similarly to spin density.

4.3. Multiconfigurational Wave Function. A wave function for the singlet state of the ozone molecule was first calculated at the HF/cc-pVDZ level. ELF and the EPLF were both calculated and give similar qualitative results (Figure 6).

Then, a complete active space wave function with eight electrons in eight orbitals (CAS $(8,8)$ ) was prepared, and EPLF was calculated (Figure 7). The EPLF obtained from the CAS wave function is 


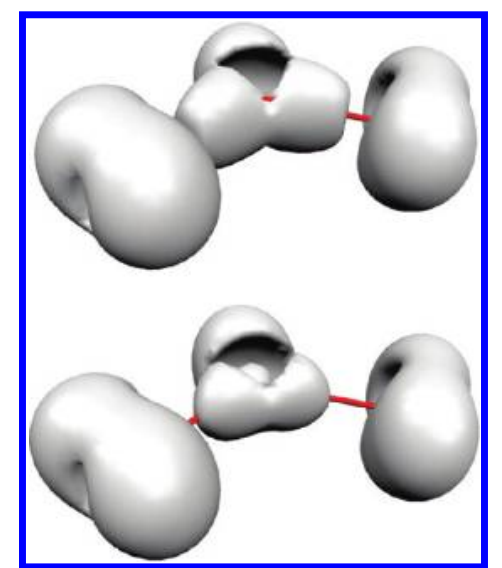

Figure 6. $\mathrm{ELF}=0.61$ (top) and EPLF $=0.123$ (bottom) isosurfaces of the singlet state of the ozone molecule (Hartree-Fock).

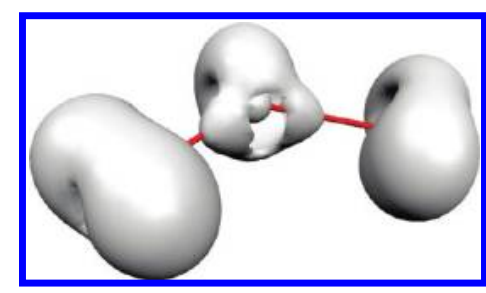

Figure 7. $\mathrm{EPLF}=0.123$ isosurface the singlet state of the ozone molecule (CAS-SCF).

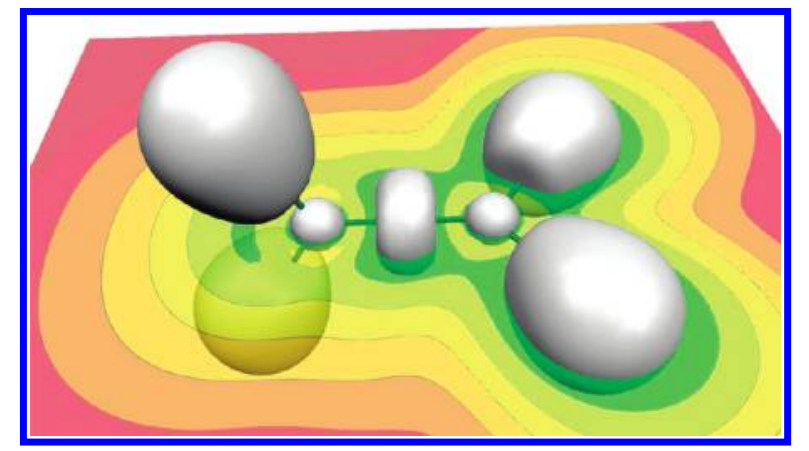

Figure 8. EPLF contour plot and isosurface of the singlet state of the twisted $\mathrm{H}_{2} \mathrm{C}^{\bullet}-{ }^{\bullet} \mathrm{CH}_{2}$ biradical. Red values of the EPLF are the lowest, and green values are the highest.

significantly different from the EPLF obtained from the HF wave function. In the $\mathrm{HF}$ framework, the $\mathrm{O}-\mathrm{O}$ bonding domains are more connected to each other than to the lone pair domains of the central oxygen atom. With the CAS wave function, each $\mathrm{O}-\mathrm{O}$ bond domain is more connected to the lone pair domains than to each other. This example shows that EPLF is an alternative to ELF in closed shell systems where a multiconfigurational method is required, as EPLF is well-defined for such cases.

4.4. Open-Shell Singlet. When the ethylene molecule is twisted with an angle of $90^{\circ}$ along the $\mathrm{C}-\mathrm{C}$ axis, the $\pi$ bond breaks. Each one of the $\pi$ electrons localizes on a carbon atom, giving rise to an open-shell singlet (see Figure 8), degenerate with the triplet state. In order to preserve the spin symmetry, a CAS $(2,2)$ wave function was computed to describe the singlet state. With such a wave function, the spin density is not able to localize the unpaired electrons since the $\alpha$ one-electron density is equal to the $\beta$ one-electron density in every point of space. The EPLF reveals the presence of these unpaired electrons by local minimum values of the function close to the carbon atoms, in the plane perpendicular to the $\mathrm{C}-\mathrm{H}$ bonds.

\section{SOFTWARE}

To realize the EPLF and ELF calculations presented in this paper, a code was written using the IRPF90 Fortran generator. ${ }^{26}$ This code is interfaced with the Gaussian 03, ${ }^{27}$ GAMESS, ${ }^{28}$ and Molpro $^{29}$ programs. As the calculation of EPLF is more expensive than the calculation of ELF, the program has been efficiently parallelized (for both EPLF and ELF calculations) using the message passing interface (MPI) library ${ }^{30}$ and exhibits a linear speedup property with the number of cores. The EPLF code is licensed under the GNU General Public License, and the source files can be downloaded from the Web at http://eplf.sourceforge.net.

\section{CONCLUSION}

In this work, we have introduced a modified version of the EPLF analytically computable for standard wave functions and DFT representations. When compared to the original EPLF defined in a QMC framework, essentially the same images are recovered. A systematic comparison of our analytical EPLF with the Electronic Localization Function (ELF) of Becke and Edgecombe has been made. For closed-shell systems, the EPLF results are shown to closely match the ELF ones. However, for other situations, the two localization functions may differ significantly (radicals, systems with strong static correlations, etc). The major advantage of the reformulated EPLF is that it can be easily computed for any kind of electronic structure method defined from single or multideterminantal wave functions. Further development will focus on the topological analysis of the EPLF, which will provide the possibility of computing various properties integrated from a partition of the three-dimensional space. As our software is available for free, it should open the possibility for any chemist to use EPLF for the understanding of complex electronic structures.

\section{ASSOCIATED CONTENT}

Supporting Information. EPLF vs ELF for the xenon atom. This material is available free of charge via the Internet at http://pubs.acs.org.

\section{AUTHOR INFORMATION}

\section{Corresponding Author}

*E-mail: scemama@irsamc.ups-tlse.fr.

\section{ACKNOWLEDGMENT}

Support from the French "Centre National de la Recherche Scientifique (CNRS)”, Université de Toulouse, and Université Paris 6 is gratefully acknowledged. We would like to thank IDRIS (CNRS, Orsay), CCRT (CEA/DAM, Ile-de-France), and CALMIP (Université de Toulouse) for providing us with computational resources.

\section{REFERENCES}

(1) Daudel, R.; Odiot, S.; Brion, H. J. Physique Rad. 1954, 15, 804809.

(2) Bader, R. F. W. Atoms in molecules: A quantum theory; Clarendon Press: Oxford, U. K., 1990; p 438. 
(3) Becke, A. D.; Edgecombe, K. E. J. Chem. Phys. 1990, 92, 53975403.

(4) Silvi, B.; Savin, A. Nature 1994, 371, 683-686.

(5) Savin, A. J. Chem. Sci. 2005, 117, 473-475.

(6) Silvi, B. J. Phys. Chem. A 2003, 107, 3081-3085.

(7) Feixas, F.; Matito, E.; Duran, M.; Solà, M.; Silvi, B. J. Chem. Theory Comput. 2010, 6, 2736-2742.

(8) Bezugly, V.; Wielgus, P.; Kohout, M.; Wagner, F. R. J. Comput. Chem. 2010, 31, 1504-1519.

(9) Cancès, E.; Keriven, R.; Lodier, F.; Savin, A. Theor. Chim. Acta 2004, 111, 373-380.

(10) Scemama, A.; Caffarel, M.; Savin, A. J. Comput. Chem. 2007, 28 (1), $442-454$.

(11) Scemama, A.; Chaquin, P.; Caffarel, M. J. Chem. Phys. 2004, 121, 1725-1735.

(12) Scemama, A.; Caffarel, M.; Ramírez-Solís, A. J. Phys. Chem. A 2009, 113, 9014-9021.

(13) Caffarel, M.; Scemama, A.; Ramírez-Solís, A. Theor. Chim. Acta 2010, 126, 275-287.

(14) Parr, R.; Yang, W. Density-Functional Theory of Atoms and Molecules; Oxford University Press: Oxford, U. K., 1989; p 344.

(15) Caffarel, M.; Hernández-Lamoneda, R.; Scemama, A.; RamírezSolís, A. Phys. Rev. Lett. 2007, 99, 153001.

(16) Amador-Bedolla, C.; Salomón-Ferrer, R.; Lester, W. A., Jr.; Vázquez-Martínez, J. A.; Aspuru-Guzik, A. J. Chem. Phys. 2007, 126, 204308 .

(17) Hammond, B. L.; Lester, W. A., Jr.; Reynolds, P. J. Monte Carlo Methods in Ab Initio Quantum Chemistry. Lecture and Course Notes in Chemistry; World Scientific: Singapore, 1994; Vol. 1.

(18) Alary, F.; Heully, J.-L.; Scemama, A.; Garreau-de Bonneval, B.; Chane-Ching, K.; Caffarel, M. Theor. Chim. Acta 2010, 126, 243-255.

(19) For a given electronic configuration $\left(\mathbf{r}_{1}, \ldots, \mathbf{r}_{N}\right), f$ goes as exp$\left(-\gamma\left|\mathbf{r}-\mathbf{r}_{\text {min }}\right|^{2}\right)$ when $\gamma$ becomes large. Accordingly, in this limit, $\langle(-\ln$ $\left.f)^{1 / 2}\right\rangle$ goes to a finite positive constant given by $\left\langle\left|\mathbf{r}-\mathbf{r}_{\min }\right|^{2}\right\rangle$. On the other hand, $\langle f\rangle$ can be written as the integral of $\exp \left(-\gamma\left|\mathbf{r}-\mathbf{r}_{\text {min }}\right|^{2}\right)$ over all possible configurations, which can be rewritten as $1 /(\gamma)^{1 / 2}(\gamma)^{1 / 2}$ $\exp \left(-\gamma\left|\mathbf{r}-\mathbf{r}_{\min }\right|^{2}\right)$, that is, at large $\gamma$, the product of $1 /(\gamma)^{1 / 2}$ times a Dirac distribution. Finally, in the large- $\gamma$ limit, the ratio $\left(\left\langle(-\ln f)^{1 / 2}\right\rangle\right) /$ $\left(\left(-\ln \langle f)^{1 / 2}\right\rangle\right)$ goes to zero as $\sim 1 /(\gamma)^{1 / 2}$.

(20) De La Lande, A.; Salahub, D. R.; Maddaluno, J.; Scemama, A.; Pilme, J.; Parisel, O.; Gerard, H.; Caffarel, M.; Piquemal, J.-P. J. Comput. Chem. 2010, [Online] DOI: 10.1002/jcc.21698.

(21) Dunning, T. H., Jr. J. Chem. Phys. 1989, 90, 1007-1023.

(22) Hariharan, P. C.; Pople, J. A. Theor. Chim. Acta 1973, 28, $213-$ 222.

(23) Francl, M. M.; Pietro, W. J.; Hehre, W. J.; Binkley, J. S.; Gordon, M. S.; DeFrees, D. J.; Pople, J. A. J. Chem. Phys. 1982, 77, 3654-3665.

(24) Becke, A. D. Phys. Rev. A 1988, 38, 3098-3100.

(25) Lee, C.; Yang, W.; Parr, R. G. Phys. Rev. B 1988, 37, 785-789.

(26) Scemama, A. ArXiv e-prints 2009, [cs.SE], 0909.5012.

(27) Frisch, M. J. et al. Gaussian 03; Gaussian, Inc.: Wallingford, CT, 2004.

(28) Schmidt, M. W.; Baldridge, K. K.; Boatz, J. A.; Elbert, S. T.; Gordon, M. S.; Jensen, J. H.; Koseki, S.; Matsunaga, N.; Nguyen, K. A.; Su, S.; Windus, T. L.; Dupuis, M.; Montgomery, J. A., Jr. J. Comput. Chem. 1993, 14 11, 1347-1363.

(29) Werner, H. J. et al. MOLPRO, version 2009.1. http://www. molpro.net (accessed Dec 7, 2009).

(30) Gropp, W.; Lusk, E.; Doss, N.; Skjellum, A. Parallel Comput. 1996, 22, 789-828. 